\title{
HIGHLY VARIABLE YOUNG MASSIVE STARS IN ATLASGAL CLUMPS
}

\author{
M. S. N. Kumar ${ }^{1,2}$, C. Contreras Peña ${ }^{1}$, P. W. LuCas $^{1}$, and M. A. Thompson ${ }^{1}$ \\ ${ }^{1}$ Centre for Astrophysics Research, University of Hertfordshire, Hatfield, AL10 9AB, UK \\ ${ }^{2}$ Instituto de Astrofísica e Ciências do Espaco, Universidade do Porto, CAUP, Rua das Estrelas, 4150-762 Porto, Portugal \\ Received 2016 June 5; revised 2016 September 22; accepted 2016 September 27; published 2016 December 5
}

\begin{abstract}
High-amplitude variability in young stellar objects (YSOs) is usually associated with episodic accretion events. It has not been observed so far in massive YSOs. Here, the high-amplitude variable star sample of Contreras Peña et al. has been used to search for highly variable ( $\Delta K \geqslant 1 \mathrm{mag}$ ) sources coinciding with dense clumps mapped using the $850 \mu \mathrm{m}$ continuum emission by the ATLASGAL survey. A total of 18 variable sources are centered on the submillimeter clump peaks and coincide $\left(<1^{\prime \prime}\right)$ with a $24 \mu \mathrm{m}$ point or compact $\left(<10^{\prime \prime}\right)$ source. Of these 18 sources, 13 can be fit by YSO models. The 13 variable YSOs (VYSOs) have luminosities of $\sim 10^{3} L_{\odot}$, an average mass of $8 M_{\odot}$, and a range of ages up to $10^{6} \mathrm{yr}$. A total of 11 of these 13 VYSOs are located in the midst of infrared dark clouds. Nine of the 13 sources have $\Delta K>2 \mathrm{mag}$, significantly higher compared to the mean variability of the entire VVV sample. The light curves of these objects sampled between 2010 and 2015 display rising, declining, or quasi-periodic behavior but no clear periodicity. Light-curve analysis using the Plavchan method shows that the most prominent phased signals have periods of a few hundred days. The nature and timescale of variations found in 6.7 Ghz methanol maser emission in massive stars are similar to that of the VYSO light curves. We argue that the origin of the observed variability is episodic accretion. We suggest that the timescale of a few hundred days may represent the frequency at which a spiraling disk feeds dense gas to the young massive star.
\end{abstract}

Key words: interstellar medium - stars: formation - stars: massive - stars: variables: T Tauri, Herbig Ae/Be

Supporting material: figure set

\section{INTRODUCTION}

Variability studies of young low-mass stars, in both the line and the continuum, have proven to be powerful tools for deciphering the physics of star formation and pre-mainsequence evolution. Low-mass young stellar objects (YSOs) displaying optical/infrared flux variability trace a variety of phenomena (Fernandez \& Eiroa 1996; Carpenter et al. 2001) such as accretion events in the disk, magnetospheric activity (Bouvier et al. 2007, pp. 479-94), spots (Bouvier \& Bertout 1989), and flares on the stellar surface. High-amplitude variability is often associated with episodic events of accretion, well known through objects such as FUors (named after FU Orionis) and EXors (named after EX Lupi). Hence, they are excellent laboratories to understand the accretion phenomenon, especially at unresolved spatial scales representing the accretion disk and the young star.

Higher-mass young stars are elusive objects that spend their brief youth while deeply embedded in extremely dense molecular cores. Optical photometric variability studies of Herbig Ae/Be stars (intermediate-mass YSOs) concluded that large-amplitude variability is confined to stars with spectral types later than B8 (Herbst \& Shevchenko 1999). This result should not be surprising, considering that stars more massive than $8 M_{\odot}$ would lack a pre-main-sequence phase appearing directly on the zero-age main sequence (Iben 1965; Hayashi 1966). In other words, optically visible intermediate and massive stars should have already arrived on the zero-agemain-sequence (Bernasconi \& Maeder 1996) with very little episodic accretion activity. Moreover, once a massive star becomes optically visible, the accretion rates are low (Hartmann et al. 1993) and the accretion luminosity itself is insignificant compared to the total luminosity. On the other hand, the infrared counterparts (IRcs) to high-mass protostellar objects (HMPOs; Kumar \& Grave 2007; Grave \& Kumar 2009) represent some sort of a pre-main-sequence phase for the massive stars because these objects are deeply embedded in their natal dense molecular cores, show high accretion rates, and are detected only in the infrared bands. Hence, they are more likely to display variability associated with accretion episodes. However, a systematic search for variability in such objects is still lacking. IRcs to HMPOs are often visible as point sources in the near-infrared $K$ band in the midst of dark clouds. The following work is an attempt to find variability in such sources.

The Vista Variables in the Via Lactea survey (VVV; Minniti et al. 2010) provides $Z Y J H K_{s}$ photometry of $\approx 560 \mathrm{deg}^{2}$ of the Galactic bulge and the adjacent midplane. In addition, the survey has yielded $\approx 50-70$ epochs of $K_{s}$ photometry over a period of $5 \mathrm{yr}$. The VVV survey covers much of the Galactic fourth quadrant, which is known for its intense activity of highmass star formation in the Milky Way. This virtue of the fourth quadrant has caused it to be the focus for several other Galactic plane surveys such as ATLASGAL (Schuller et al. 2009) and HiGal (Molinari et al. 2010), aiming to uncover deeply embedded high-mass stars and cluster-forming cores. The ATLASGAL survey has provided an unbiased data set of dense molecular clumps. These are massive clumps, often associated with high-mass star formation. Prior to the VVV study, no eruptive variable YSOs (VYSOs) were known with luminosities higher than a few hundred solar luminosities (Audard et al. 2014). In this work we inspect the VVV sample of 816 high-amplitude infrared variable stars discovered by Contreras et al. (2016a), and we isolate variables that coincide with ATLASGAL clumps in an effort to find variable young intermediate/high-mass stars. 
Table 1

Source Details

\begin{tabular}{|c|c|c|c|c|c|c|c|}
\hline VYSO & $\begin{array}{l}\text { R.A. } \\
\text { (deg) }\end{array}$ & $\begin{array}{l}\text { Decl. } \\
\text { (deg) }\end{array}$ & $\begin{array}{c}K_{s}^{\mathrm{a}} \\
(\mathrm{mag})\end{array}$ & $\begin{array}{l}\Delta K_{s}^{\mathrm{b}} \\
(\mathrm{mag})\end{array}$ & $\begin{array}{l}\sigma K_{s}^{\mathrm{c}} \\
(\mathrm{mag})\end{array}$ & $\begin{array}{l}\text { Light-curve } \\
\text { Type }^{\mathrm{d}}\end{array}$ & Association \\
\hline VVVv244 & 245.00828 & -51.43392 & 14.4 & 2.08 & 0.59 & LPV-YSO & IRDC \\
\hline VVVv263 & 245.43405 & -50.34484 & 13.9 & 2.24 & 0.64 & Eruptive & IRDC \\
\hline VVVv367 & 255.12338 & -43.88343 & 14.5 & 2.35 & 0.67 & Fader & IRDC \\
\hline VVVv374 & 254.64164 & -42.83201 & 11.7 & 2.41 & 0.73 & Eruptive & IRDC, EGO, $\mathrm{rms}^{\mathrm{e}}$ \\
\hline VVVv389 & 255.82157 & -42.43052 & 14.3 & 1.56 & 0.41 & Fader & IRDC \\
\hline VVVv665 & 242.49040 & -50.80262 & 13.4 & 1.63 & 0.43 & Eruptive & IRDC \\
\hline VVVv717 & 249.02318 & -46.67795 & 12.5 & 2.47 & 0.64 & LPV-YSO & IRDC \\
\hline VVVv736 & 252.73104 & -44.11650 & 15.5 & 1.52 & 0.25 & Dipper & IRDC \\
\hline VVVv750 & 253.18580 & -43.08889 & 15.8 & 2.73 & 0.82 & STV & IRDC \\
\hline VVVv802 & 258.54427 & -38.50329 & 13.2 & 1.42 & 0.40 & LPV-Mira & IRDC \\
\hline
\end{tabular}

Notes.

${ }^{a}$ Average magnitudes.

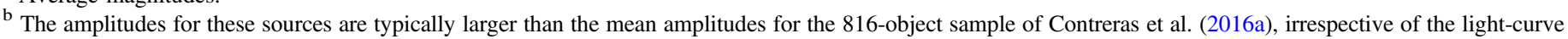
classification.

${ }^{\mathrm{c}}$ rms variation of $\Delta K_{s}$ as a function of $K_{s}$ for the given VVV tile.

${ }^{\mathrm{d}} \mathrm{LPV}=$ long periodic variable; STV $=$ short timescale variable.

${ }^{\mathrm{e}} \mathrm{rms}=\operatorname{red} M S X$ source (Lumsden et al. 2013).

\section{HIGH-AMPLITUDE VARIABLE STARS WITHIN ATLASGAL CLUMPS}

The sample of high-amplitude ( $\Delta K_{s}>1 \mathrm{mag}$ ) variable stars uncovered from the VVV survey has been described by Contreras et al. (2016a). They found 816 variable stars in the 4th Galactic quadrant $\left(l=295^{\circ}\right.$ to $350^{\circ}, b=-1^{\circ} .1$ to $\left.1^{\circ} .0\right)$. The identification method and the selection criteria are detailed in Contreras et al. (2016a). Here, the interest is to find variable stars that are IRcs to HMPOs. By definition an HMPO should be associated with a massive molecular clump, typical of the ATLASGAL clumps. The 816 high-amplitude variable stars were matched with a radius of $300^{\prime \prime}$ to all the peak positions listed in the ATLASGAL web database, resulting in 39 matched sources. To ensure that the matched variable star actually represents a luminous (massive) source with infrared excess (young star), rather than an unrelated foreground or background source, we used the following constraints in the next step of filtering. The ATLASGAL data have an angular resolution of $19 ! \prime 2$ and a pointing rms better than $4^{\prime \prime}$. The variable star associated with the submillimeter clump (almost always within the peak of the 19 !' 2 beam) must coincide (to better than $1^{\prime \prime}$ ) with a $24 \mu \mathrm{m}$ source that is either point or compact $\left(<10^{\prime \prime}\right)$ like. A total of 18 of the 39 stars above satisfied these criteria. Next, the spectral energy distribution (SED) of the source should be representative of a YSO and not a stellar photosphere. Therefore, the $1-850 \mu \mathrm{m}$ photometric measurements for these 18 objects were assembled using any, and all available data from the 2MASS, GLIMPSE, MIPSGAL, WISE, HiGal, and ATLASGAL databases (see Section 3 and Table 2). The resulting SEDs were fitted using the SED fitting tool described by Robitaille et al. (2007), which attempts to model the observed SED with an appropriate YSO model, at the same time checking whether the SED can represent a mainsequence photosphere. Five of the 18 objects were fitted by stellar photospheres, leaving $13 \mathrm{VVV}$ sources that were fit with
YSO models. These 13 VYSOs are listed in Table 1, along with their known properties.

In Table $1, \sigma K_{s}$ is the typical scatter in each magnitude bin of $K_{s}$ for a given VVV tile (see Contreras et al. (2016a) for a full description). This scatter is in the range of $0.4-0.6 \mathrm{mag}$, as is typical with uncorrected pipeline photometry of the VVV tiles. The choice of $\Delta K_{s}>1$ mag cutoff (in both this work and that of Contreras et al. 2016a) is a conservative choice to select objects well above the scatter $\sigma K_{s}$. This choice is the reason why only a small number of 13 VYSOs are found in this study. An extended study is underway to examine the general variability characteristics of high-mass YSOs by employing the relatively accurate, paw-print photometry that displays lower scatter levels.

To visualize and examine the environment of the VYSOs, we composed color images using the Spitzer MIPSGAL (Carey et al. 2009) and GLIMPSE (Churchwell et al. 2009) cutouts and overlaid contours of $850 \mu \mathrm{m}$ emission from the ATLASGAL data. In Figure 1 (left panel) we display such an overlay for the source VVVv374. It is an excellent example of the 13 objects listed in Table 1. The Spitzer MIPS $24 \mu \mathrm{m}$, IRAC $8 \mu \mathrm{m}$, and IRAC $3.6 \mu \mathrm{m}$ images are red, green, and blue, respectively, and the yellow contours show the ATLASGAL $850 \mu \mathrm{m}$ emission. Similar plots for the remaining 12 targets are shown in the supplementary Figure Set 1 . In Figure 1 (left panel) the infrared VYSO appears as a bright $24 \mu \mathrm{m}$ source, embedded inside the elliptical-shaped dense core traced by the $850 \mu \mathrm{m}$ emission contours. The dark patch in the background image reveals the associated infrared dark cloud. In 11 of the 13 cases, the ATLASGAL cores are associated with a cataloged infrared dark cloud (IRDC; Peretto \& Fuller 2009). The presence of an IRDC is an important signpost that the selected objects are indeed sites of massive star formation. The star formation activity in VVVv374 is further evident through the presence of an extremely green object (EGO; Cyganowski et al. 2008). The right panel in Figure 1 shows this feature for 


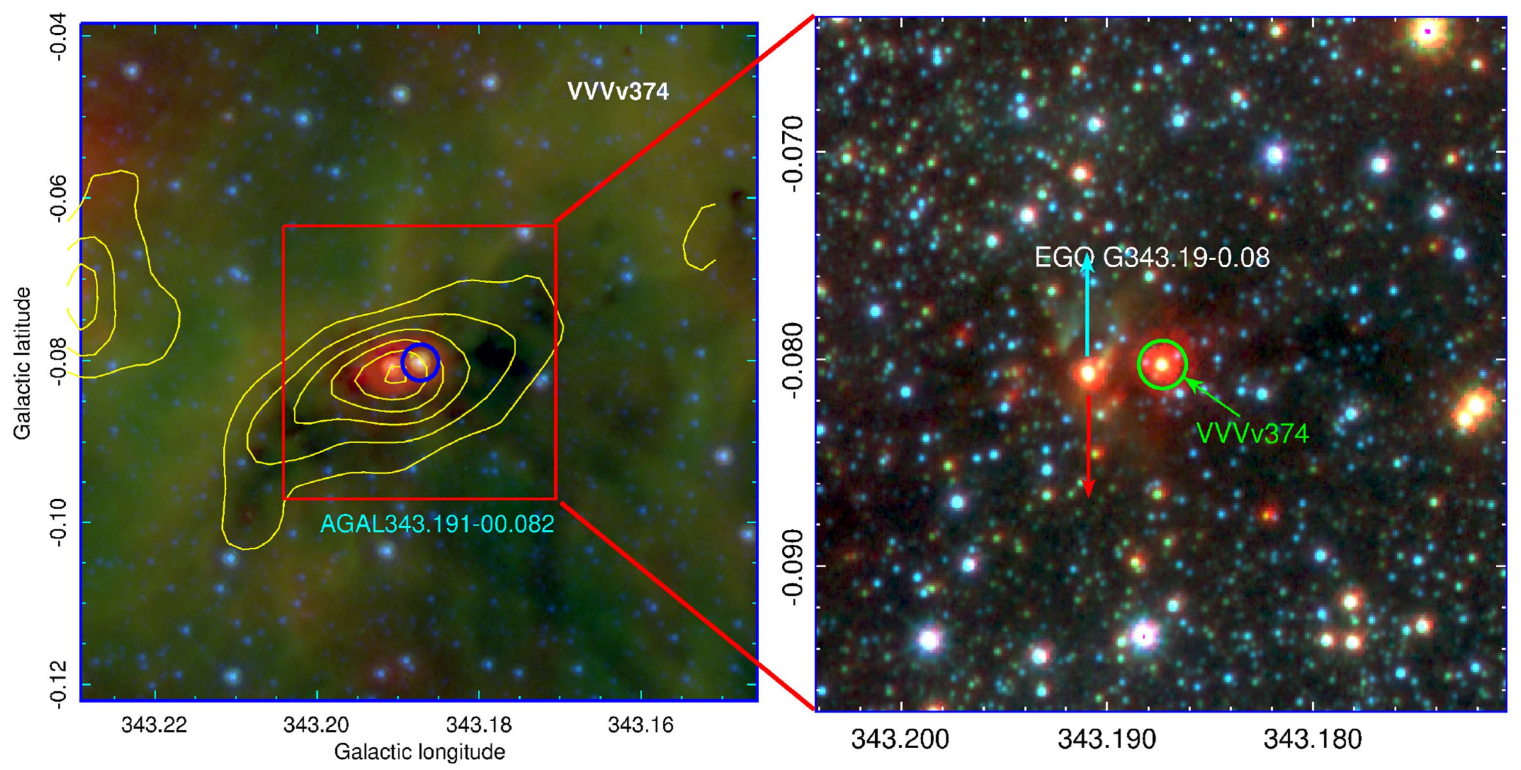

Figure 1. The environment of VVVv374. Left Panel: Spitzer-MIPS $24 \mu \mathrm{m}$, IRAC $8 \mu \mathrm{m}$ and $3.6 \mu \mathrm{m}$ images are coded as red, green and blue respectively to compose the colour image which reveal the infrared dark filament/cloud. Yellow contours show the $870 \mu \mathrm{m}$ emission (starting at $0.1 \mathrm{Jy}$, in steps of $0.1 \mathrm{Jy}$ ) from the ATLASGAL data tracing the molecular clump associated with the infrared dark cloud. Two young stars are visible at the peak of the clump. The blue circle identifies the variable young star. Right Panel: Zoom in view of the two young stars shown with a colour composite of the Spitzer-IRAC $4.5 \mu \mathrm{m} \mathrm{VVV} \mathrm{K}_{s}$ and VVV H-band images coded as red, green and blue respectively. The young star adjacent to the variable source is associated with an extended green object (EGO) as shown. Blue and red arrows represent possible blue and red shifted lobes of the outflow associated with the EGO.

(The complete figure set ( 2 images) is available.)

VVVv374, highlighting the bipolar outflow traced by the EGO. Here the IRAC $4.5 \mu \mathrm{m}$ and VVV $K$ - and $H$-band images are used as red, green, and blue respectively. The Vizier database was used to search a $30^{\prime \prime}$ radius around each target to find known signposts of star formation such as IRDCs, EGOs, masers, and HII regions, and they are listed in the last column of Table 1.

We note that $9 / 13$ sources in Table 1 have amplitudes $\Delta K_{s}>2 \mathrm{mag}$, which is significantly higher than the mean variability of all the YSOs in the VVV sample of Contreras et al. (2016a), regardless of the light-curve classification. In that study, high amplitude was found to be correlated with an early stage of evolution, as traced by the 2-22 $\mu \mathrm{m}$ spectral index. The presence of ATLASGAL cores and IRDCs tends to support this correlation, in addition to indicating that highamplitude VYSOs can be massive systems.

\section{SED FITTING}

By using the SED modeling, one can qualitatively assess the luminosity and mass of the stars of interest. The photometric data compiled for the 13 objects are tabulated in Table 2. It includes the four Spitzer IRAC bands from the GLIMPSE survey $(3.6 \mu \mathrm{m}, 4.5 \mu \mathrm{m}, 5.8 \mu \mathrm{m}, 8 \mu \mathrm{m})$, two bands from the WISE survey $(12 \mu \mathrm{m}, 22 \mu \mathrm{m})$, Spitzer MIPS photometry at the $24 \mu \mathrm{m}$ band (Robitaille et al. 2008), five bands from the Herschel-HiGal survey $(70 \mu \mathrm{m}, 170 \mu \mathrm{m}, 250 \mu \mathrm{m}, 350 \mu \mathrm{m}$, $500 \mu \mathrm{m}$ ), and the ATLASGAL $850 \mu \mathrm{m}$ band. Whenever MIPS $24 \mu \mathrm{m}$ photometry is available, the WISE $22 \mu \mathrm{m}$ is ignored to ensure using the higher-quality data. Given that our targets display $>1$ mag variation in the $K$ band and the above surveys are noncontemporaneous, the variations in the near-infrared $J$ and $H$ bands are expected to be of similar amplitude. Therefore, they are not used to model the SEDs. In the IRAC $3.6 \mu \mathrm{m}$ and $4.5 \mu \mathrm{m}$ bands, typically $0.2-0.4$ mag variations are reported for low-mass YSOs (Faesi et al. 2012; Wolk et al. 2015). Moreover, Contreras et al. (2016a) typically find lower-amplitude variability at these longer wavelengths compared to $K$-band variation. Such variations should not affect the overall SED and the resulting fit.

The photometric data listed in Table 2 were fed to the SED fitting tool (Robitaille et al. 2007). The physics of YSOs behind this tool is detailed in Robitaille et al. (2006). We used appropriate apertures for each band, a classification as data or upper limit, and a range in distance and extinction for each source. The aperture diameters used are $4^{\prime \prime}$ for Spitzer IRAC, $12^{\prime \prime}$ for WISE bands, and $6^{\prime \prime}$ for MIPS $24 \mu \mathrm{m}$. The FWHM of the HiGal and ATLASGAL cores were used as aperture diameters for the submillimeter data.

The data at wavelengths shorter than (and inclusive of) $24 \mu \mathrm{m}$ were employed as "data points" (as they critically define the fitted models), and the remaining longer-wavelength data are set as "upper limits" (which allow us to constrain the fitted models) in the SED fitting (e.g., Grave \& Kumar 2009). This choice is made to minimize the contamination from other sources enclosed within the large beams of the longwavelength data. The SED fitting tool scales and fits YSO models derived from a library of 200,000 models. The scaling is done by adopting an appropriate combination of extinction and distance within a range provided by the user. Neither of these factors is known with certainty (see Contreras et al. 2016a) for our sources, so we used a fixed range of $A_{v}=5-50 \mathrm{mag}$ and $d=1-13 \mathrm{kpc}$ for all 13 objects. Typically, the adopted choice of the SED fitter is correct within a factor of two for both values because of the logical combination of YSO models stored in the library. The choice of $A_{v}$ and the distance used by the SED fitter is an output from the fitting results, listed in Table 3 along with other parameters. We note that IRDCs in quadrant 4 are typically located at distances of $\sim 3 \mathrm{kpc}$ (Jackson et al. 2008), and distances over $\sim 8 \mathrm{kpc}$ are unlikely because 
Table 2

Photometric Data Used for SED Fitting

\begin{tabular}{|c|c|c|c|c|c|c|c|c|c|c|c|c|c|}
\hline VYSO & $\begin{array}{c}\mathrm{I} 1 \\
(\mathrm{mag})\end{array}$ & $\begin{array}{c}\mathrm{I} 2 \\
(\mathrm{mag})\end{array}$ & $\begin{array}{c}\mathrm{I} 3 \\
(\mathrm{mag})\end{array}$ & $\begin{array}{c}\mathrm{I} 4 \\
(\mathrm{mag})\end{array}$ & $\begin{array}{c}\text { W3 } \\
\text { (mag) }\end{array}$ & $\begin{array}{c}\text { W4 } \\
\text { (mag) }\end{array}$ & $\begin{array}{c}\text { M1 } \\
\text { (mag) }\end{array}$ & $\begin{array}{l}\text { PACS70 } \\
\text { (Jy) }\end{array}$ & $\begin{array}{c}\text { PACS160 } \\
\text { (Jy) }\end{array}$ & $\begin{array}{l}\text { SPIRE250 } \\
(\mathrm{Jy})\end{array}$ & $\begin{array}{c}\text { SPIRE350 } \\
\text { (Jy) }\end{array}$ & $\begin{array}{c}\text { SPIRE500 } \\
(\mathrm{Jy})\end{array}$ & $\begin{array}{l}\text { AGAL850 } \\
\text { (Jy) }\end{array}$ \\
\hline VVVv244 & 11.2 & 10.0 & 8.8 & 8.2 & 6.0 & 4.1 & $\ldots$ & 2.4 & 10.73 & 20.23 & 12.88 & 7.42 & 0.84 \\
\hline VVVv263 & 9.6 & 8.1 & 6.8 & 6.0 & 5.40 & $2.3^{\mathrm{a}}$ & 1.9 & 3.63 & 17.23 & $\ldots$ & $\ldots$ & $\ldots$ & 15.0 \\
\hline VVVv367 & 9.8 & 8.3 & 7.0 & 5.8 & 4.6 & 1.9 & $\ldots$ & 6.05 & 5.75 & 7.75 & $\ldots$ & $\ldots$ & 4.00 \\
\hline VVVv374 & 7.4 & 6.5 & 5.6 & 4.8 & 3.9 & 1.7 & $\ldots$ & 40.52 & 35.98 & 65.92 & 40.79 & 25.93 & 3.00 \\
\hline VVVv389 & 10.0 & 8.7 & 7.9 & 7.3 & 6.2 & 3.6 & $\ldots$ & 0.84 & 5.10 & 12.99 & 15.49 & 38.74 & 13.42 \\
\hline VVVv665 & 10.0 & 8.9 & 7.8 & 7.0 & 6.3 & $3.8^{\mathrm{a}}$ & 3.7 & 0.94 & 18.46 & $\ldots$ & $\ldots$ & $\ldots$ & 1.89 \\
\hline VVVv717 & 10.1 & 8.8 & 7.6 & 6.8 & 6.5 & $4.4^{\mathrm{a}}$ & 4.0 & 0.57 & 2.08 & $\ldots$ & $\ldots$ & $\ldots$ & 2.45 \\
\hline VVVv736 & 10.6 & 9.4 & 8.0 & 7.3 & 5.7 & $2.9^{\mathrm{a}}$ & 4.5 & 67.71 & 71.60 & 71.79 & 47.11 & 28.77 & 1.21 \\
\hline VVVv750 & 12.4 & $\ldots$ & 9.6 & 9.0 & 6.3 & 2.0 & $\ldots$ & 20.69 & 15.56 & 21.45 & 17.50 & 9.80 & 1.89 \\
\hline VVVv802 & 8.2 & 6.5 & 5.4 & 4.7 & 4.2 & 1.7 & $\ldots$ & 31.11 & 13.97 & 20.31 & 14.87 & 14.10 & 3.71 \\
\hline
\end{tabular}

Note.

${ }^{\mathrm{a}}$ Not used.

these dense clouds require a bright mid-infrared background in order to be observed in silhouette. The SED fitting results are consistent with the expected locations on the near side of the Milky Way.

The fitting procedure is based on a $\chi^{2}$ minimization method and yields a best-fit model, together with a range of models satisfying that the criterion $\chi_{\text {best }}^{2}-\chi^{2}$ per data point is less than 3. The model parameters listed in Table 3 are weighted means of this suite of models, the weights being the inverse of the $\chi^{2}$ of each model. In Table 3 the column $N_{\text {fits }}$ indicate the number of models used to compute the parameters satisfying the above criteria, and $N_{\text {fits } 2}$ are the number of models that have a nonzero value of envelope accretion rate. The fitted YSO models for the exemplar source VVVv374 are shown in Figure 2. The remaining fits are displayed in the supplementary Figure Set 2.

\subsection{SED Fitting Results}

The most reliable output of the SED fitting procedure is the luminosity because it are obtained by a simple scaling of the precomputed radiation transfer models to match the flux peak and shape of the observed SED by using an appropriate combination of extinction and distance to minimize the $\chi^{2}$ (Robitaille et al. 2007). This is also the reason why the extinction and distance estimates given by the fitting procedure turn out to be correct within a factor of two. This procedure will select optimal combinations of mass and age to match the observed SED from a uniformly sampled parameter space (see Robitaille et al. 2006), which is used to derive the temperature and radius of the young star through evolutionary models. From Table 3 it can be seen that the selected high-amplitude variable stars are best modeled as intermediate- to high-mass objects with an average luminosity of $10^{3} L_{\odot}$ and mass of $8 M_{\odot}$.

The modeled ages show that they are typically million-yearold objects, with a significant level of disk and envelope accretion rates. The number of models $N_{\text {fits2 }}$ with a nonzero envelope accretion rate is smaller compared to the total number of models, because much of the longer-wavelength data are used as upper limits, to avoid contribution from adjacent sources. However, in $9 / 13$ sources the HiGal $70 \mu \mathrm{m}$ datum corresponds to an unresolved source detected within $5^{\prime \prime}$ of the
VVV source. Since the Herschel/PACS beam size is $6^{\prime \prime} \times 12^{\prime \prime}$ at this wavelength, this strongly implies that the $70 \mu \mathrm{m}$ flux arises in the disk or envelope of the YSO, rather than the largerscale cloud. If we were to use the $70 \mu \mathrm{m}$ data for these nine sources actively in the fit, instead of upper limits, at least $6 / 13$ sources would be fitted with a nonzero envelope accretion rate, compared to the $2 / 13$ sources in Table 3 . Consequently, it appears likely that these YSOs have a range of ages extending below $10^{6}$ yr. We also note that using HiGal fluxes as data points instead of upper limits would tend to increase the total luminosity of these systems.

The 13 targets are all near-infrared visible, suggesting that they have partially emerged from the deeply embedded phase, which is often captured only at the submillimeter regime. The inferred disk accretion rates represent a mean value of $10^{-6} M_{\odot} \mathrm{yr}^{-1}$. This is two orders of magnitude smaller than the high disk accretion rates measured through millimeter observations (Cesaroni et al. 2007) or from SED modeling of IRcs to HMPOs (Grave \& Kumar 2009). The fitting results can be improved by acquiring higher spatial resolution data at longer wavelengths. The results obtained here are merely indicative; the main inference is that the selected targets represent YSOs of high bolometric luminosity, therefore representing massive YSOs.

\section{LIGHT CURVES}

The VVV data obtained between 2010 and 2015 have been used to construct the light curves for the 13 sources here. The light curve from the multi-epoch photometric data for the source VVVv374 is shown in Figure 3. The remaining light curves are displayed in the supplementary Figure Set 3. It can be seen that the data points are spread out fairly uniformly along the time axis with several gaps in between, as is typical for the survey observations. The gaps are often larger than 1 week between consecutive observations, but shorter, intraday time intervals are also sampled. The time series will nearly always detect changes in flux that endure for several months (Contreras et al. 2016a). Regular variations with periods from hours to weeks are also likely to be detected, though one-off variations on these short timescales are likely to be missed. The light-curve classifications for these objects from Contreras et al. 
Table 3

SED Fitting Results

\begin{tabular}{|c|c|c|c|c|c|c|c|c|c|c|c|}
\hline VYSO & $\begin{array}{c}M_{*} \\
\left(M_{\odot}\right)\end{array}$ & $\begin{array}{l}A_{V, \text { core }} \\
(\mathrm{mag})\end{array}$ & $\begin{array}{l}A_{V, \text { int }} \\
\text { (mag) }\end{array}$ & $\left(10^{-5} \dot{M}_{\odot} \mathrm{yr}^{-1}\right)$ & $\begin{array}{c}\dot{M}_{\mathrm{disc}} \\
\left(10^{-6} \mathrm{M}_{\odot} \mathrm{yr}^{-1}\right)\end{array}$ & $\underset{(y r)}{\log A g e}$ & $\begin{array}{l}\text { Distance } \\
(\mathrm{kpc})\end{array}$ & $\begin{array}{c}\log L_{\text {tot }} \\
\left(L_{\odot}\right)\end{array}$ & $\chi_{\text {best }}^{2} / N_{\text {data }}$ & $N_{\text {fits }}$ & $\overline{N_{\text {fits2 }}}{ }^{a}$ \\
\hline VVVv244 & $5.9 \pm 2.6$ & $192 \pm 4009$ & $32 \pm 13$ & $7.6 \pm 26.2$ & $1.1 \pm 6.0$ & $6.2 \pm 0.8$ & $5.5 \pm 3.5$ & $2.8 \pm 0.7$ & 0.05 & 10000 & 1392 \\
\hline VVVv263 & $8.5 \pm 2.8$ & $97 \pm 595$ & $48 \pm 7$ & $3.3 \pm 3.5$ & $3.8 \pm 27.0$ & $6.2 \pm 0.7$ & $4.2 \pm 2.6$ & $3.5 \pm 0.5$ & 0.65 & 684 & 49 \\
\hline VVVv336 & $7.3 \pm 2.9$ & $206 \pm 2951$ & $43 \pm 9$ & $0.0 \pm 0.0$ & $0.7 \pm 2.8$ & $6.4 \pm 0.2$ & $5.7 \pm 3.3$ & $3.2 \pm 0.6$ & 0.09 & 3333 & 8 \\
\hline VVVv367 & $8.3 \pm 3.7$ & $641 \pm 3857$ & $31 \pm 14$ & $3.8 \pm 4.2$ & $8.7 \pm 34.0$ & $5.7 \pm 1.2$ & $4.9 \pm 2.9$ & $3.3 \pm 0.6$ & 0.16 & 1035 & 363 \\
\hline VVVv374 & $8.8 \pm 2.7$ & $121 \pm 1480$ & $15 \pm 4$ & $1.6 \pm 7.9$ & $0.6 \pm 4.2$ & $6.3 \pm 0.2$ & $4.4 \pm 2.5$ & $3.5 \pm 0.4$ & 0.14 & 1659 & 113 \\
\hline VVVv389 & $7.3 \pm 2.5$ & $67 \pm 1185$ & $37 \pm 9$ & $0.4 \pm 0.9$ & $0.7 \pm 3.1$ & $6.4 \pm 0.3$ & $5.1 \pm 3.1$ & $3.2 \pm 0.5$ & 0.38 & 3200 & 75 \\
\hline VVVv405 & $11.1 \pm 3.3$ & $340 \pm 3631$ & $45 \pm 6$ & $0.0 \pm 0.0$ & $1.8 \pm 6.3$ & $6.3 \pm 0.2$ & $4.4 \pm 2.3$ & $3.8 \pm 0.4$ & 0.64 & 806 & 0 \\
\hline VVVv406 & $5.3 \pm 2.5$ & $83 \pm 3391$ & $13 \pm 11$ & $2.8 \pm 3.2$ & $2.8 \pm 8.7$ & $4.9 \pm 0.9$ & $5.7 \pm 3.6$ & $2.6 \pm 0.7$ & 0.25 & 3172 & 2812 \\
\hline VVVv665 & $7.6 \pm 2.5$ & $69 \pm 1056$ & $37 \pm 8$ & $0.0 \pm 0.2$ & $0.7 \pm 2.7$ & $6.4 \pm 0.2$ & $5.5 \pm 3.2$ & $3.3 \pm 0.5$ & 0.03 & 3969 & 51 \\
\hline VVVv717 & $8.5 \pm 2.8$ & $73 \pm 691$ & $44 \pm 6$ & $0.0 \pm 0.0$ & $1.5 \pm 4.1$ & $6.3 \pm 0.2$ & $5.7 \pm 3.3$ & $3.5 \pm 0.5$ & 0.49 & 1459 & 0 \\
\hline VVVv736 & $7.5 \pm 2.9$ & $224 \pm 3415$ & $36 \pm 12$ & $58.8 \pm 96.1$ & $0.5 \pm 3.5$ & $6.4 \pm 0.3$ & $5.9 \pm 3.3$ & $3.2 \pm 0.6$ & 0.0 & 5515 & 204 \\
\hline VVVv750 & $7.4 \pm 3.3$ & $450716 \pm 5232901$ & $11 \pm 8$ & $15.8 \pm 17.0$ & $19.8 \pm 71.2$ & $4.1 \pm 0.7$ & $6.2 \pm 3.8$ & $2.9 \pm 0.6$ & 0.08 & 1807 & 1806 \\
\hline VVVv802 & $11.5 \pm 3.2$ & $160 \pm 2169$ & $47 \pm 5$ & $0.9 \pm 1.7$ & $2.3 \pm 10.0$ & $6.3 \pm 0.2$ & $3.5 \pm 1.6$ & $3.9 \pm 0.4$ & 0.29 & 1635 & 5 \\
\hline
\end{tabular}

Note.

${ }^{\mathrm{a}}$ The number of models with a nonzero envelope accretion rate. 


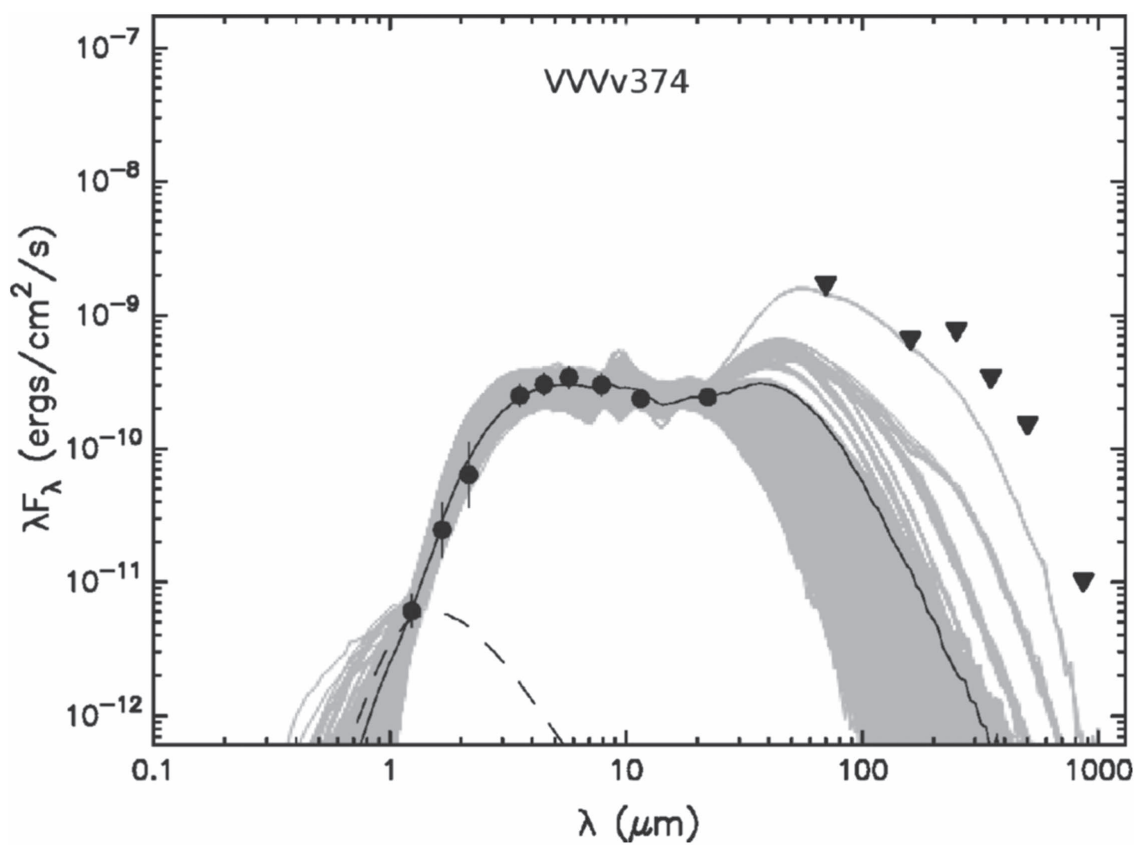

Figure 2. SED fitting results for the source VVVv374. Filled circles and triangles show the "data points" and "upper limits" respectively. Solid black line and grey lines show the best fit model and the models satisfying the criteria $\chi_{\text {best }}^{2}-\chi^{2}<3$ respectively. The dashed line represents the embedded photosphere of the best fit YSO model.

(The complete figure set (1 images) is available.)

(2016a), based on the 2010-2015 time series, are listed in Table 1.

Examining the light curves of all 13 VYSOs, we do not find visually obvious periodicity, except in the case of VVVv802. This light curve was classified as LPV-Mira by Contreras et al. (2016a), owing to the excellent fit to a sine curve in the 2010-2014 time series. However, the 2015 datum departs from the previous trend. Moreover, the SED of this source (especially the 24 and $70 \mu \mathrm{m}$ data) does not resemble those of dusty Mira variables discussed by Contreras et al. (2016b), so the Mira interpretation is disfavored. The observed light curve is the sum of flux variations caused by a variety of phenomena, such as accretion, spots, eruptive events, and lineof-sight extinction due to rotation of the source (Carpenter et al. 2001). The light curves of some pre-main-sequence stars are shown to have contributions from pulsations (Kallinger et al. 2008). Additionally, noise from variable seeing and weather conditions also contributes to the observed light curve. Analyzing the light curve with a periodogram can often uncover a phased signal if it happens to be of substantial significance.

To carry out such analysis, we used the NASA exoplanet archive periodogram tool. ${ }^{3}$ This tool offers various algorithms to process both periodic (such as exoplanet or asteroseismic signals) and nonperiodic time-series data. The Plavchan method (Plavchan et al. 2008) is most suited to examine nonperiodic light-curve data. This algorithm uses a phasedispersion minimization method to detect phased signals by minimizing the residuals to phase-folded light curves with a range of possible periods. In using the Plavchan algorithm, the user can adjust values of the number of outlier data points and

\footnotetext{
3 http://exoplanetarchive.ipac.caltech.edu/applications/Periodogram/docs/ Algorithms.html
}

the phase-smoothing box size. These parameters were left at default values of 500 and 0.060 , which would allow us the liberty to reject all data points (maximum 50 for the data here) if no suitable signal was found. On ingesting the light curves to the periodogram tool, it attempts to detect and list phased signals of different frequencies. The signal power is the magnitude of the coefficients in the frequency domain, and the probability of obtaining the calculated power for a particular period by chance is given by a $p$-value. The output is organized to list the most significant periods first, i.e., those with the highest signal power and least $p$-value. The $p$-values are in the range between 0 and 1 . Of interest here is to detect potential phased signals contributing to the light curve. Therefore, we selected the first three significant periods, which displayed the least $p$-value, in most cases about four to six orders of magnitude smaller than the $p$-values of subsequent periods. In other words, the first two or three significant periods (with the highest powers) displayed $p$-values of the order of $10^{-6}-10^{-9}$, and the subsequent $p$-values were of the order of $10^{-1}-10^{-3}$ or larger. The most significant periods are listed in Table 4, where, for each VYSO, the columns Per1, Per2, and Per3 show the periods and the columns Power1, Power2, and Power3 are the respective signal powers. If only two periods Per1 and Per2 are listed for a given source, it means that the power spectrum is relatively clean, lacking further components.

From Table 4 it is evident that the most significant period (Per1) is of the order of a few hundred days. The median values of Per1, Per2, and Per3 for the 13 sources are 492, 284, and 124 days, respectively. The Plavchan method is known to be particularly sensitive in producing false peaks at integer multiples of the fundamental period of a real signal, so we compared Per1, Per2, and Per3 for every source in Table 4. There are only limited signs of such an artifact here: some of 


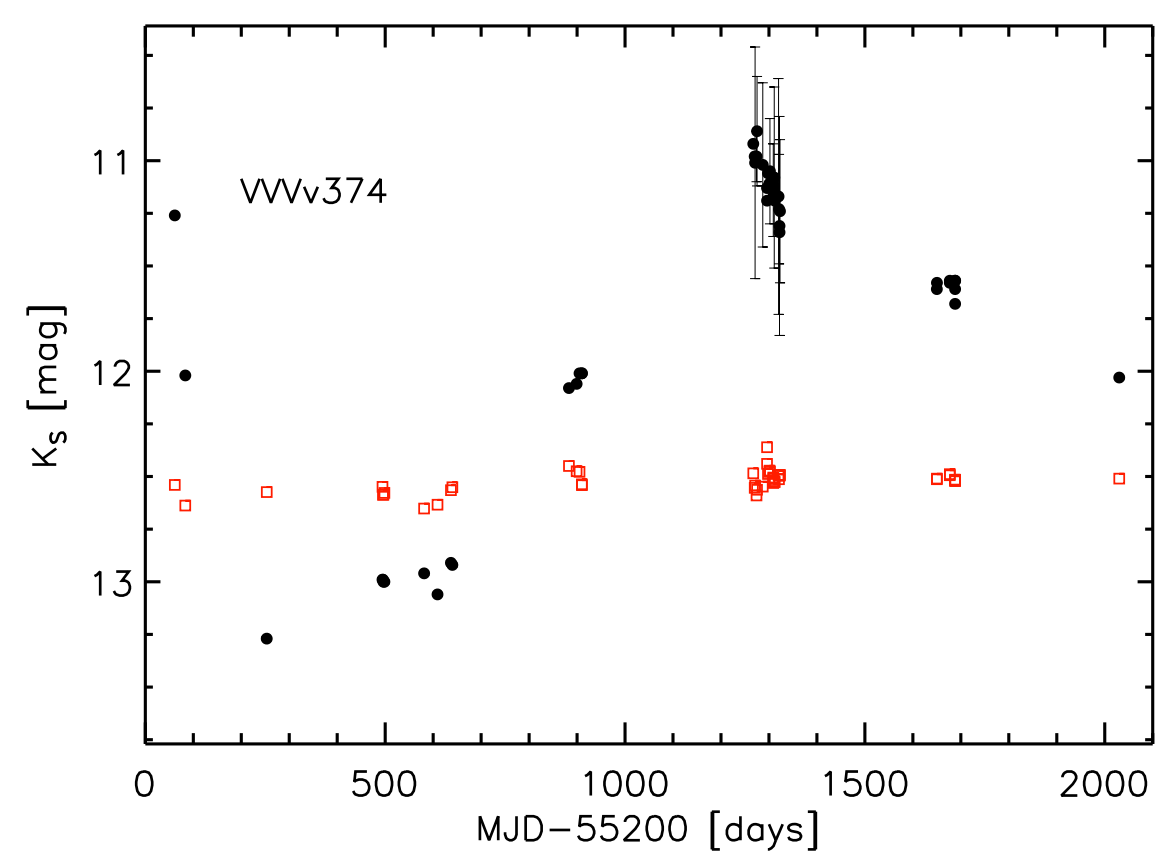

Figure 3. Light curve of the source VVVv374. Red squares show the light curve of a non-varying control object within the same field of view.

(The complete figure set (1 images) is available.)

the periods of the sources VVVv367, VVVv374, and VVVv405 are close to harmonics.

\section{NEAR-INFRARED COLORS}

The $J H K$ color properties of the 13 VYSOs are examined. Multiband $J H K$ data are available for two epochs, roughly at the beginning and the end of the available light-curve baseline. In Figure 4 we show these two epochs on the $J-H$ versus $H-K$ color-color diagram. The first and last epoch are represented by red and blue symbols, respectively, joined by a black line. Only three VYSOs are detected in all the three $J H K$ bands at both epochs. The remaining VYSOs have missing detections, either in the $J, H$, or both, in one or both epochs, owing to the very red nature of these sources. In such cases we have used the VVV sensitivity limits as an upper limit in computing the colors, and they are indicated by the arrows. The VYSOs occupy the reddened HAeBe locus (Lada \& Adams 1992) of the color-color space. The HAeBe locus encompasses intermediate-massive disk $\left(L_{\text {disk }}=0.1 L_{\text {bol }}\right)$ objects with central holes at $T_{D} \geqslant 1000 \mathrm{~K}$. The 13 VYSOs are reddened by different amounts, as evident from the vertical distribution of their location parallel to the reddening vector. In the $5 \mathrm{yr}$ time span, the VYSOs have mostly remained in the HAeBe space, with some variations in reddening. This may suggest that infrared excess variations are the cause of photometric variability, although the lack of detections in all three bands may imply variable reddening as a possible origin.

\section{DISCUSSION}

In all but one source, the VYSO is offset by $<5^{\prime \prime}$ of the ATLASGAL peak. The $850 \mu \mathrm{m}$ peak itself is often associated with a red diffuse (VVVv736, VVVv406) or point source (VVVv374, VVVv336, VVVv367). In the exemplary target VVVv374, the red point source coinciding with the $850 \mu \mathrm{m}$ peak is found to drive an EGO. This feature, that the VYSO is located adjacent to a younger and/or more embedded YSO, may point to a physical association between the two, with some evolutionary lag, the VYSO having formed first. Numerical simulations of high-mass star formation (ex Krumholz et al. 2009) predict such pairs, the younger companion forming due to fragmentation of a Jeans unstable massive disk/envelope. In VVVv263, though the $850 \mu \mathrm{m}$ emission appears diffuse, a high-contrast filamentary dark cloud is located in the midst of the emission, and the VYSO is centered at the peak of this infrared filament.

The masses of all 13 VYSOs listed in Table 3 are in the range 5-11 $M_{\odot}$, mostly of 7-8 $M_{\odot}$, representing massive YSO candidates. The distances (from SED models) are in the range of $3.5-6.5 \mathrm{kpc}$, and the internal core extinction is a hundred to a few hundred visual magnitudes. Contreras et al. (2016b) have estimated the distance to five of these VYSOs based on radial velocities and/or association with star-forming regions. The SED modeled distances are roughly in agreement with those measurements to better than a factor of two. The models fitted to the source VVVv750 yield an abnormal $A_{v}$ of $450,716 \mathrm{mag}$, and it is the only target with a very young age estimate. This VYSO is associated with one of the most circular and isolated ATLASGAL cores, and its light curve is also the most jittery (fluctuating), standing out from the rest.

Large-amplitude variability in YSOs can arise due to episodic accretion events, varying extinction due to orbital motion of dusty clumps or a warped disk, or eclipsing binary or multiple sources. Spots, flares, and pulsations can produce only low-amplitude variability. The nonperiodic nature of the VYSO light curves rules out the possibility of eclipsing near-equalmass objects. High-mass star formation is well known to produce multiplicity, even at the formation stages (due to fragmentation), resulting in near-equal-mass objects and/or several low-mass objects. The light curves for the sample here are only sensitive to near-equal-mass companions. Varying extinction if it occurs should reflect in an associated reddening 


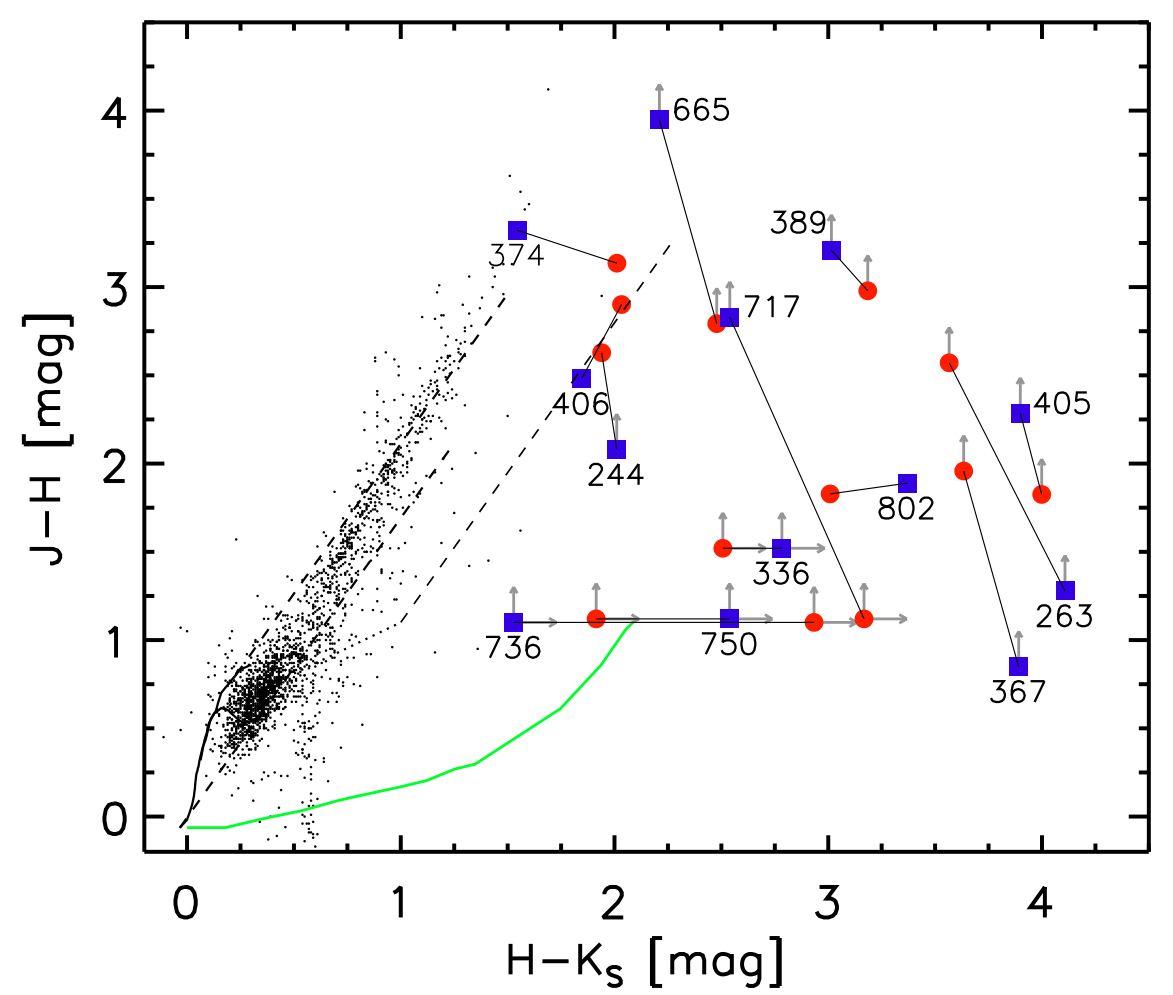

Figure 4. Color-color diagram. Black dots are the typical near-infrared colors of stars in a $6^{\prime} \times 6^{\prime}$ field centered on the variable sources. The dwarf and giant locus are shown with thick black lines. The dotted line shows the T Tauri locus. Dashed lines enclosing these curves are reddening vectors isolating the reddened groups of those objects. The green curve displays the HAeBe locus. The red and blue data points display the colors of each object at the beginning and end of the light curve.

Table 4

Light-curve Analysis Results

\begin{tabular}{lrrrrcc}
\hline \hline VYSO & Per1 $^{\text {a }}$ & Pwr1 $^{\text {b }}$ & Per2 & Pwr2 & Per3 & Pwr3 \\
\hline VVVv244 & 107.7 & 28.5 & 809.0 & 26.0 & 7.0 & 24.7 \\
VVVv263 & 314.0 & 122.7 & 560.0 & 54.3 & $\ldots$ & $\ldots$ \\
VVVv336 & 886.3 & 20.8 & 283.5 & 20.1 & 327.1 & 9.5 \\
VVVv367 & 289.7 & 14.8 & 146.9 & 10.7 & 114.0 & 8.5 \\
VVVv374 & 454.0 & 59.1 & 251.3 & 22.4 & 124.3 & 12.1 \\
VVVv389 & 188.5 & 12.2 & 233.1 & 12.2 & 438.7 & 11.5 \\
VVVv405 & 491.5 & 12.4 & 231.1 & 6.5 & 113.4 & 6.5 \\
VVVv406 & 491.9 & 227.1 & 939.0 & 66.6 & $\ldots$ & $\ldots$ \\
VVVv665 & 834.7 & 23.1 & 303.9 & 7.2 & $\ldots$ & $\ldots$ \\
VVVv717 & 851.0 & 177.8 & 277.4 & 68.8 & $\ldots$ & $\ldots$ \\
VVVv736 & 493.1 & 18.8 & 311.5 & 13.3 & 235.6 & 9.5 \\
VVVv750 & 66.2 & 183.9 & 44.4 & 169.2 & $\cdots$ & $\cdots$ \\
VVVv802 & 631.4 & 326.7 & 890.7 & 165.4 & $\cdots$ & $\ldots$ \\
\hline
\end{tabular}

Notes. Sources with missing p3 display a relatively clean power spectrum with two significant periods of probability statistics $p$-value $=0$.

${ }^{a}$ Period in days.

${ }^{b}$ Power of Per1.

of the source. The 13 VYSOs are included in the larger sample of Contreras et al. (2016a), who have examined the reddening effects and find that it is not a dominant mechanism to reproduce the high-amplitude variability. Therefore, the most likely origin of the observed large-amplitude variability in these intermediate- to high-mass VYSOs must be accretion events. Intermediate-resolution near-infrared spectra for five of the VYSOs $(374,405,406,665,717)$ have been discussed by Contreras et al. (2016b). All five sources display shocked $\mathrm{H}_{2}$ emission at $2.12 \mu \mathrm{m}$, irrespective of their light-curve classification. Three of these sources also display $\mathrm{Br} \gamma$ in emission. Both $\mathrm{H}_{2}$ and $\mathrm{Br} \gamma$ emission are consistent with the scenario of ongoing accretion. The case of V723 Carinae as a massive eruptive variable (Tapia et al. 2015) published during the course of this study represents another excellent case of the variable phenomenon in massive young stars.

The analysis of the light curves showed that the most significant underlying phased signals have periods of the order of a few hundred days with a median of $\sim 500$ days. This timescale and the nature of variability found here (eruptive, dipper, LPV) are very similar to the variations of the $6.7 \mathrm{GHz}$ class II methanol maser emission in high-mass star-forming regions, both in its nature (Goedhart et al. 2004) and in the timescales (Goedhart et al. 2014). The class II methanol masers are thought to be pumped by infrared radiation (Cragg et al. 2005) and found to originate in accretion disks in highmass star-forming regions (Sanna et al. 2010; Sugiyama et al. 2014). The similarity in the variability found in the methanol maser emission (MME) and the IR variability may therefore represent the frequency of the infrared luminosity variations of the warm disk from accretion events, which is also responsible for pumping the class II MME (Cragg et al. 2005). We searched for MME in the 13 VYSOs and did not find any methanol masers within a radius of $30^{\prime \prime}$. De Villiers et al. (2015) argue that even though the onset of the $6.7 \mathrm{GHz}$ MME is strongly associated with the outflow, and hence accretion activity, a critical abundance of the methanol molecule is required to produce the MME. It is therefore likely that the MME sources are younger and/or embedded in denser regions in comparison with the evolutionary states of the VYSOs studied here. Nevertheless, the common origin of both MME and IR variability is strongly associated with the accretion 
phenomenon, in which case the inferred timescale of a few hundred days may represent the timescale at which density enhancements of a spiraling disk feeds the central young star.

\section{CONCLUSIONS}

This study has found hitherto unknown near-infrared variability in intermediate- to high-mass young stars by using the VISTA VVV data from 2010-2015. Following a stringent selection criterion to select targets with $\Delta K \geqslant 1$ mag, 13 VYSOs located at the peak of ATLASGAL clumps are identified. These sources are characterized by modeling their $1-850 \mu \mathrm{m}$ SEDs and by analyzing their light curves with a phase-dispersion minimization method.

1. The SED modeling of the 13 VYSOs shows that their luminosities are $\sim 10^{3} L_{\odot}$, the stellar masses are in the range of $8-11 M_{\odot}$, and the ages are in the range of $10^{4}$ $10^{6} \mathrm{yr}$.

2. The light curves are not periodic in nature. They can be classified as rising, declining, or quasi-periodic. Analysis using the Plavchan method reveals that the most prominent underlying periodic signal would have an average period of $\sim 500$ days.

3. The high-amplitude variability in young massive stars is attributed to episodic accretion events.

M.S.N.K. is supported through H2020 Marie-Curie IntraEuropean Fellowship project GESTATE (661249). This work was supported by the UK's Science and Technology Facilities Council, grant numbers ST/M001008/1 ST/L001403/1 and $\mathrm{ST} / \mathrm{J} 001333 / 1$. We gratefully acknowledge the use of data from the ESO Public Survey program 179.B-2002 taken with the VISTA $4.1 \mathrm{~m}$ telescope and data products from the Cambridge Astronomical Survey Unit. This research has made use of the SIMBAD database, operated at CDS, Strasbourg, France; also the SAO/NASA Astrophysics data (ADS). C.C.P. was supported by a University of Hertfordshire $\mathrm{PhD}$ studentship in the earlier stages of this research.

\section{REFERENCES}

Audard, M., Abraham, P., Dunham, M. M., et al. 2014, in Protostars and Planets VI, ed. H. Beuther et al. (Tucson, AZ: Univ. Arizona Press), 387 Bernasconi, P. A., \& Maeder, A. 1996, A\&A, 307, 829
Bouvier, J., Alencar, S. H. P., Harries, T. J., Johns-Krull, C. M., \& Romanova, M. M. 2007, in Protostars \& Planets V, ed. B. Reipurth, D. Jewitt, \& K. Keil (Tucson, AZ: Univ. Arizona Press)

Bouvier, J., \& Bertout, C. 1989, A\&A, 211, 99

Carey, S. J., Noriega-Crespo, A., Mizuno, D. R., et al. 2009, PASP, 121, 76

Carpenter, J. M., Hillenbrand, L. A., \& Skrutskie, M. E. 2001, AJ, 121, 3160

Cesaroni, R., Galli, D., Lodato, G., Walmsley, C. M., \& Zhang, Q. 2007, in Protostars and Planets V, ed. B. Reipurth, D. Jewitt, \& K. Keil (Tucson, AZ: Univ. Arizona Press), 197

Churchwell, E., Babler, B. L., Meade, M. R., et al. 2009, PASP, 121, 213

Contreras, C., Lucas, P. W., Minniti, D., et al. 2016a, MNRAS, in press (arXiv:1602.06267)

Contreras, C., Lucas, P. W., Minniti, D., et al. 2016b, MNRAS, in press (arXiv:1602.06269)

Cragg, D. M., Sobolev, A. M., \& Godfrey, P. D. 2005, MNRAS, 360, 533

Cyganowski, C. J., Whitney, B. A., Holden, E., et al. 2008, AJ, 136, 2391

de Villiers, H. M., Chrysostomou, A., Thompson, M. A., et al. 2015, MNRAS, 449, 119

Faesi, C. M., Covey, K. R., \& Gutermuth, R. 2012, PASP, 124, 1137

Fernandez, M., \& Eiroa, C. 1996, A\&A, 310, 143

Goedhart, S., Gaylard, M. J., \& van der Walt, D. J. 2004, MNRAS, 355, 553

Goedhart, S., Maswanganye, J. P., Gaylard, M. J., \& van der Walt, D. J. 2014, MNRAS, 437, 1808

Grave, J. M. C., \& Kumar, M. S. N. 2009, A\&A, 498, 147

Hartmann, L., Kenyon, S. J., \& Calvet, N. 1993, ApJ, 407, 219

Hayashi, C. 1966, ARA\&A, 4, 171

Herbst, W., \& Shevchenko, V. S. 1999, AJ, 118, 1043

Iben, I. 1965, ApJ, 141, 993

Jackson, J. M., Susanna, C., Rathborne, J. M., Chambers, E. T., \& Simon, R. 2008, ApJ, 680, 349

Kallinger, T., Zwintz, K., \& Weiss, W. 2008, A\&A, 488, 279

Krumholz, M. R., Klein, R. I., McKee, C. F., Offner, S. R., \& Cunningham, A. J. 2009, Sci, 323, 754

Kumar, M. S. N., \& Grave, J. M. C. 2007, A\&A, 462, 37

Lada, C. J., \& Adams, F. C. 1992, ApJ, 393, 278

Lumsden, S., Hoare, M. G., Urquhart, J. S., et al. 2013, ApJS, 208, 11

Minniti, D., Lucas, P. W., Emerson, J. P., et al. 2010, NewA, 15, 433

Molinari, S., Swinyard, B., Bally, J., et al. 2010, PASP, 122, 314

Peretto, N., \& Fuller, G. A. 2009, A\&A, 505, 405

Plavchan, P., Kirkpatrick, J. D., Cutri, R. M., \& Gallagher, S. C. 2008, ApJS, 175,191

Robitaille, T. P., Meade, M. R., Babler, B. L., et al. 2008, AJ, 136, 2413

Robitaille, T. P., Whitney, B. A., Indebetouw, R., \& Wood, K. 2007, ApJS, 169,328

Robitaille, T. P., Whitney, B. A., Indebetouw, R., Wood, K., \& Denzmore, P. 2006, ApJS, 167, 256

Sanna, A., Moscadelli, L., Cesaroni, R., et al. 2010, A\&A, 517, 78

Schuller, F., Menten, K. M., Contreras, Y., et al. 2009, A\&A, 504, 415

Sugiyama, K., Fujisawa, K., Doi, A., et al. 2014, A\&A, 562, 82

Tapia, M., Roth, M., \& Persi, P. 2015, MNRAS, 446, 4088

Wolk, S. J., Günther, H. M., Poppenhaeger, K., et al. 2015, AJ, 150, 145 